\title{
L'impatto farmacoeconomico del trattamento della schizofrenia con antipsicotici tipici ed atipici: l'esperienza di un DSM della Regione Sicilia
}

\author{
Tommaso Federico*, Carmelo Astuto*, Giuseppe Bongiorno*, Domenico Majorana*, \\ Giovanni Biricolti§, Salvatore Zerbo*
}

Il presente lavoro è stato realizzato con il patrocinio del Dipartimento Ispettorato Regionale Sanitario dell'Assessorato per la Sanità della Regione Sicilia, nella persona del Dirigente Generale Dott. Saverio Ciriminna

\begin{abstract}
BACKGROUND: The comparatively high acquisition costs of the new antipsychotic drugs have induced the mental health community to look closely at their potential benefits.

OBJECTIVE: To compare the clinical and economic outcomes associated with olanzapine, risperidone and typical neuroleptics treatment for schizophrenia.

METHODS: A multicenter, observational, two-years long, retrospective and prospective study was conducted with 229 psychotic patients (in charge by psychiatric Centers of Regione Sicilia - Italy). Clinical outcomes were assessed using changes in CGI (Clinical Global Impression) and PANSS (Positive and Negative Syndrome Scale) scores. The economic data collection included pharmacological and non-pharmacological resources consumption (hospitalizations, medical/nurse visits, etc.). The economic evaluation was conducted in the perspective of the Local Psychiatric Services.

RESULTS: The results in terms of clinical performance indicated an advantage (statistically significant) in the olanzapine group of patients. The pharmacological costs were significantly lower $(p<0,05)$ in the typical group due to the cheaper acquisition cost of these drugs while total treatment costs were similar between the three groups ( $p>0,05)$. Treatment with olanzapine was associated with a lower non-pharmacological resources consumption and showed a general reduction ( $\mathrm{p}<0,05$ vs. risperidone) of total treatment costs between 1 st and 2nd year of observation.

CONCLUSIONS: Within the context of the local health care Services, olanzapine appears to be the "dominant" therapeutic option compared with risperidone and typical neuroleptics. Treatment with risperidone appears to be "cost-neutral" compared with typical neuroleptics.
\end{abstract}

Keywords: olanzapine, risperidone, typical neuroleptics, schizophrenia, clinical and economic outcomes Farmeconomia e percorsi terapeutici 2005; 6 (4): 365-375

\section{INTRODUZIONE}

La schizofrenia rappresenta una patologia psichiatrica devastante e costosa, di complessa gestione per gli Operatori sanitari [1]. Ad essa si associa generalmente una seria compromissione clinica, sociale e lavorativa per chi ne soffre nonché un pesante carico per i familiari del paziente e un rilevante onere economico per il Sistema Sanitario [2,3].

La riduzione dei contatti sociali, l'appiattimento affettivo, la disorganizzazione, la mancanza di motivazioni e le difficoltà cognitive sono i sintomi negativi più spesso presenti in questa malattia. A questi si possono accom- pagnare sintomi positivi quali aggressività, tentativi di suicidio, violenze su persone o cose. Tali episodi, sicuramente i più evidenti e socialmente compromettenti, richiedono quasi sempre periodi di ricovero ospedaliero e successivamente il ricorso a strutture riabilitative di media e lungodegenza [4]. Questo aspetto fa si che gli investimenti per l'assistenza e la cura del paziente schizofrenico incidano con una quota rilevante sulla spesa sanitaria [5].

Intorno ai primi anni '90 sono stati introdotti sul mercato gli antipsicotici “atipici”, una nuova classe di farmaci che ha fatto registra- 


\begin{tabular}{|c|c|c|c|c|}
\hline \multirow{5}{*}{ 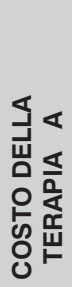 } & \multicolumn{4}{|c|}{ EFFICACIA DELLA TERAPIA A } \\
\hline & & $<\mathrm{B}$ & $=\mathrm{B}$ & $>\mathrm{B}$ \\
\hline & $<\mathrm{B}$ & $?$ & SI & $\mathrm{SI}$ \\
\hline & $=\mathrm{B}$ & NO & $?$ & $\mathrm{SI}$ \\
\hline & $>\mathrm{B}$ & NO & NO & ? \\
\hline
\end{tabular}

Figura 1

Schema per analisi decisionale: metriche di confronto/scelta della terapia $\mathrm{A}$ rispetto alla terapia $B$ rapie antipsicotiche più efficienti che consentano una ottimale allocazione delle risorse sanitarie.

A tal riguardo si è condotta un' analisi su pazienti psicotici in carico presso il Dipartimento di Salute Mentale della ASL 3 di Catania focalizzando il confronto farmacoeconomico fra il trattamento con i tradizionali neurolettici tipici e quello con i due atipici a maggior diffusione (olanzapina e risperidone).

\section{OBIETTIVO}

L'obiettivo principale di questo studio è quello di valutare e confrontare costi ed efficacia associati a due anni di trattamento di pazienti schizofrenici in regime di terapia con antipsicotici tipici ed atipici (olanzapina e risperidone) ed in carico presso tre moduli dipartimentali (CT1, CT2 e CT3) del DSM (Dipartimento di Salute Mentale) della ASL 3 di Catania.

L' eventuale cambiamento delle condizioni cliniche e generali dei pazienti (relative anche al comportamento, alle relazioni sociali e alle condizioni di vita) nel periodo di osservazione dello studio non è dovuto, ovviamente, solo al farmaco somministrato, ma anche agli interventi psicoterapeutici e riabilitativi. Tuttavia, il confronto avviene sotto l'ipotesi che, a parità di efficacia degli altri trattamenti sanitari, le differenze nei risultati siano attribuibili al tipo di farmaco impiegato [4].

Gli antipsicotici atipici sono caratterizzati da un costo di acquisto significativamente più alto dei neurolettici tipici [8]. Nell'ambito del presente lavoro desideriamo tuttavia spostare l'attenzione dal semplice costo di acquisizione dei farmaci al costo globale di trattamento del paziente psicotico che include altre rilevanti voci di spesa per il Sistema Sanitario quali le prestazioni psichiatriche territoriali e le ospedalizzazioni rese necessarie durante le fasi di riacutizzazione della patologia.

L'analisi è stata condotta nella prospettiva della ASL ed in particolare delle Strutture erogatrici delle prestazioni psichiatriche. A tal riguardo la valorizzazione delle risorse sanitarie consumate dai pazienti psicotici ha fatto riferimento ai costi di produzione/erogazione dei trattamenti da parte delle Strutture Sanitarie Pubbliche $[4,9]$, nonché ai soli prezzi dei farmaci a carico del SSN.

\section{IL CONTESTO DI ANALISI}

Ai fini di questa valutazione farmacoeconomica sono stati considerati 229 pazienti afferenti al DSM della ASL 3 di Catania, con diagnosi prevalente di schizofrenia e consumo di antipsicotici tipici o atipici.

Il DSM della ASL 3 di Catania, che conta attualmente circa 16.000 utenti, si articola, al- 
l'interno del territorio provinciale, in Moduli dipartimentali ciascuno dei quali dispone al suo interno delle seguenti Strutture [10] :

- CSM (Centro di Salute Mentale): centro organizzativo dell'attività di tutela della salute mentale che svolge funzioni di coordinamento delle attività psichiatriche sul territorio;

- ambulatori distrettuali;

- SPDC (Servizio Psichiatrico di Diagnosi e Cura): strutture ospedaliere in cui vengono assicurati i ricoveri per pazienti psichiatrici;

- DH (Day Hospital): struttura diurna con finalità clinico-riabilitative annessa all'SPDC;

- CD (Centro Diurno): struttura diurna con finalità socio-riabilitative;

- CTA (Comunità Terapeutica Assistita): struttura residenziale psichiatrica con funzioni terapeutico-riabilitative.

\section{MATERIALIE METODI}

Questo lavoro basa la propria valutazione economica su dati che provengono da uno studio osservazionale multicentrico della durata di due anni condotto su 229 pazienti affetti da disturbi psicotici (in gran parte schizofrenici), in trattamento con farmaci tipici ed atipici e seguiti da tre Moduli dipartimentali (CT1, CT2 e CT3) del DSM della ASL 3 di Catania.

La scelta di procedere attraverso uno studio osservazionale è stata dettata dall'esigenza di ottenere una valutazione economica del trattamento della schizofrenia il più possibile aderente alla reale pratica clinica seguita all'interno delle Strutture psichiatriche.

L'osservazione sui pazienti è stata condotta in senso retrospettivo per 12 mesi (d'ora in poi anno I) raccogliendo dati relativi alle prestazioni psichiatriche territoriali, ai ricoveri e al consumo di farmaci impiegati per il trattamento con olanzapina, risperidone o neurolettici tipici di pazienti psicotici in carico presso il DSM catanese.

Gli stessi pazienti, al termine della fase retrospettiva, sono stati seguiti, in senso prospettico, per altri 12 mesi (d'ora in poi anno II) ove, oltre a raccogliere gli stessi dati di assorbimento di risorse sanitarie, si è proceduto a valutare gli outcome clinici associati alle diverse terapie antipsicotiche attraverso la somministrazione delle scale di valutazione psichiatrica CGI (Clinical Global Impression) e PANSS (Positive and Negative Syndrome Scale) [11]. La CGI è una scala generica di valutazione dell'efficacia e sicurezza di un trattamento psichiatrico mentre la PANSS è costituita da due sottoscale che registrano il livello di gravità dei sintomi po-

\begin{tabular}{llcc}
\hline Gruppo di diagnosi & \multicolumn{1}{c}{ Diagnosi secondo ICD-10 } & $\mathbf{N}^{\circ}$ & $\%$ \\
\hline Paranoid schizophrenia & paranoid schizophrenia (F20.0) & 86 & $100 \%$ \\
Others schizophrenia & hebephrenic schiziphrenia (F20.1) & 16 & $22,5 \%$ \\
& $\begin{array}{l}\text { residual schizophrenia (F20.5) } \\
\text { schizoaffective disorder (F25.9) }\end{array}$ & 8 & $11,3 \%$ \\
& $\begin{array}{l}\text { schizotypal disorder (F21) } \\
\text { undifferentiated schizophrenia }\end{array}$ & 5 & $72,3 \%$ \\
Psychotic disorders & $\begin{array}{l}\text { Acute and transient psychotic } \\
\text { disorder, unspecified (F23.9) }\end{array}$ & 7 & $16,9,9 \%$ \\
& $\begin{array}{l}\text { Delusional disorder (F22.0) } \\
\text { Unspecified non-organic psychosis } \\
\text { disorder (F29) }\end{array}$ & 11 & $154,3 \%$ \\
\hline Totale & $75,0 \%$ \\
\hline
\end{tabular}

Tabella I

Tipologia di diagnosi osservate e relativa numerosità all'interno del campione

sitivi (positive subscale) e negativi (negative subscale) in popolazioni affette da psicosi. I test di valutazione psichiatrica sono stati somministrati ai pazienti alla baseline (d'ora in poi T0) in coincidenza con l'inizio dell'anno II e ad un successivo follow-up dopo 12 mesi (d'ora in poi Tf), in coincidenza con la fine dell'anno II.

L'osservazione sull'intero campione di pazienti si è svolta in un intervallo di tempo tra gli anni 2003 e 2005, comprendendo sia la fase retrospettiva che quella prospettica.

Sono stati arruolati pazienti affetti da disturbi psicotici (diagnosi confermata secondo ICD-10) e trattati con neurolettici tipici (52 pazienti), olanzapina (102 pazienti) e risperidone (75 pazienti). L'inclusione dei pazienti nei tre gruppi (olanzapina, risperidone, neurolettici tipici) è stata guidata dalla terapia antipsicotica principale che, come requisito di arruolamento, doveva essere prescritta per tutto l'anno I e da almeno tre mesi prima di questo.

Il campione osservato è costituito da 137 maschi e 92 femmine con un'età media di 42 anni (range 20-74).

Le diagnosi, osservate secondo la classificazione ICD-10 [12], sono state quelle riportate in Tabella I.

La Tabella II evidenzia invece il tipo di trattamento attuato in relazione alle diagnosi rilevate nello studio.

I pazienti osservati erano in trattamento naturalistico e sono stati inseriti nei tre gruppi secondo l'antipsicotico principale di cura e rispettando l'equi-gravità. Per soddisfare questo parametro si è utilizzato il "giudizio clinico" espresso dai sanitari locali circa la gravità della patologia psicotica al momento dell'inizio della fase retrospettiva. La Ta- 


\begin{tabular}{lllc}
\hline \multicolumn{1}{c}{ Gruppo di diagnosi } & \multicolumn{1}{c}{ Trattamento } & $\mathbf{N}^{\circ}$ & $\%$ \\
\hline Paranoid schizophrenia & olanzapina & 38 & $44,2 \%$ \\
& risperidone & 36 & $41,9 \%$ \\
& neurolettici tipici & 12 & $14,0 \%$ \\
& totale & 86 & $100 \%$ \\
Others schizophrenia & olanzapina & 29 & $40,8 \%$ \\
& risperidone & 22 & $31,0 \%$ \\
Psychotic disorders & neurolettici tipici & 20 & $28,2 \%$ \\
& totale & 71 & $100 \%$ \\
& olanzapina & 35 & $48,6 \%$ \\
& risperidone & 17 & $23,6 \%$ \\
& neurolettici tipici & 20 & $27,8 \%$ \\
& totale & 72 & $100 \%$ \\
\hline
\end{tabular}

\section{Tabella II}

Tipologia di trattamento attuato in relazione alla diagnosi rilevata

\begin{tabular}{lccc}
\hline \multicolumn{1}{c}{ Giudizio clinico } & Olanzapina & Risperidone & Tipici \\
\hline Patologia psichiatrica limite & $0 \%$ & $0 \%$ & $0 \%$ \\
Patologia psichiatrica moderata & $24,3 \%$ & $28,0 \%$ & $30,4 \%$ \\
Patologia psichiatrica grave & $64,5 \%$ & $65,9 \%$ & $67,8 \%$ \\
Patologia psichiatrica molto grave & $11,2 \%$ & $6,1 \%$ & $1,8 \%$ \\
Patologia psichiatrica gravissima & $0 \%$ & $0 \%$ & $0 \%$ \\
Totale & $100 \%$ & $100 \%$ & $100 \%$ \\
\hline
\end{tabular}

Tabella III

Distribuzione dei pazienti per gravità della patologia all'interno di ciascun gruppo di trattamento

\section{IDATI RACCOLTI}

Nel corso del periodo di osservazione sono stati raccolti dati riguardanti sia l'efficacia che i costi di trattamento associati alle tre terapie antipsicotiche confrontate.

Da un punto di vista economico sono stati rilevati i costi medici diretti ovvero il consumo da parte del paziente psicotico di tutte le prestazioni sanitarie attribuibili direttamente alla patologia $[13,14]$. In tal senso sono stati raccolti, in entrambi gli anni di osservazione, dati sui consumi dei farmaci per il trattamento della patologia schizofrenica (antipsicotici principale ed associati, antidepressivi, anticolinergici, stabilizzatori dell'umore), sulle ospedalizzazioni e sull'utilizzo di servizi psichiatrici territoriali (visite specialistiche, interventi medici domiciliari, interventi infermieristici domiciliari e in struttura, interventi di psicoterapia e riabilitazione, frequenza dei centri diurni).

In linea con il punto di vista adottato per questa analisi, la valorizzazione dei consumi di servizi sanitari da parte dei pazienti è stata effettuata facendo riferimento ai costi reali di erogazione delle prestazioni sostenuti all'interno delle Strutture Psichiatriche Pubbliche. Tali valori di costo sono stati tratti da alcune recenti pubblicazioni e attualizzati all'anno 2005 $[9,4]$. La valutazione dei costi ospedalieri è stata effettuata sulla base del numero dei giorni di ospedalizzazione rilevati per paziente, valorizzati attraverso i dati di costo per giornata di ricovero in SPDC (Servizio Psichiatrico di Diagnosi e Cura) [4].

I consumi farmacologici sono stati valorizzati utilizzando i prezzi al pubblico al lordo dello sconto SSN riportati dall'Informatore farmaceutico Italiano [15]

L'efficacia clinica dei trattamenti è stata rilevata soltanto nel corso dell'anno II di osservazione, non essendo disponibili per l'anno I dati di "outcome" rilevati attraverso l'uso di scale di valutazione psichiatrica.

In sede di valutazione prospettica (anno II) i risultati associati alle diverse terapie sono stati ottenuti con la somministrazione ai pazienti delle scale CGI (Clinical Global Impression) e PANSS (Positive and Negative Syndrome Scale). Tali rilevazioni sono state effettuate alla baseline (T0-inizio anno II) e ad un successivo follow-up dopo 12 mesi (Tf-fine anno II).

\section{RISULTATI} te delle formulazioni orali ad assunzione quotidiana.

Questo aspetto ha determinato una numerosità del gruppo "tipici" più bassa rispetto ai gruppi degli "atipici” in considerazione dell'alta percentuale di pazienti in terapia con neurolettici depot presso il DSM catanese.
Analisi dell'efficacia di trattamento

La valutazione dei risultati di efficacia attraverso la scala CGI ha evidenziato un leggero aumento del punteggio medio di gravità, nel corso dell'anno II, nel gruppo trattato con tipici mentre riduzioni di gravità si sono 
riscontrate nei due gruppi di atipici con una significatività statistica rilevabile nel solo gruppo olanzapina $(\mathrm{p}<0,001)$ (Tabella IV). Tale gruppo mantiene una significativa riduzione del punteggio CGI anche verso il gruppo di trattamento con risperidone $(\mathrm{p}=0,0018)(\mathrm{Ta}-$ bella IV).

La somministrazione ai pazienti della scala CGI ci ha permesso di raggruppare i pazienti in due cluster [16]: alta gravità (marcata, grave, molto grave) e bassa gravità (molto lieve, lieve, moderata). Nel cluster "alta gravità" al T0 si rilevavano le seguenti percentuali di pazienti per gruppo di trattamento: olanzapina $47 \%$, risperidone $53 \%$, tipici $33 \%$; questa invece la situazione al T0 nel cluster "bassa gravità": olanzapina 53\%, risperidone 47\%, tipici 67\%.

Al follow-up finale dello studio (Tf) si rilevava la seguente situazione circa la percentuale di pazienti passati dalla fascia "alta gravità" alla fascia "bassa gravità": olanzapina $12 \%$, risperidone $0 \%$, tipici $+5 \%$ (Figura 2 ).

Infine la valutazione attraverso l'item CGI "Indice di efficacia" ci ha permesso di rilevare la seguente situazione nei tre gruppi circa la percentuale di pazienti che, al termine dell'anno II, ha evidenziato un effetto terapeutico da moderato (sensibile miglioramento remissone parziale dei sintomi) a notevole (netto miglioramento - remissione completa o quasi di tutti i sintomi) senza riscontrare alcun effetto collaterale: olanzapina $43 \%$, risperidone $27 \%$, tipici $29 \%$ (Figura 3 ).

La somministrazione della PANSS ha fatto registrare invece riduzioni medie di gravità statisticamente significative (tra T0 e TF) su tutti e tre i gruppi di trattamento e con riferimento a tutti i sotto-item della scala (eccezion fatta per i tipici sulla scala positiva). Il confronto fra $i$ tre gruppi in termini di riduzione media di punteggio di gravità fra T0 e Tf non ha fatto rilevare differenze statisticamente significative (Tabella V).

\section{Analisi dei costi}

Gli antipsicotici atipici, a fronte di una efficacia superiore rispetto ai tipici nel ridurre $\mathrm{i}$ sintomi della schizofrenia e nel determinare minori effetti collaterali severi, presentano un costo di acquisizione significativamente superiore [17].

Questo dato viene confermato dalla Tabella VI che evidenzia i dati sui costi farmacologici medi annui a paziente per gruppo di terapia ove la voce "Totale farmaci" fa rilevare differenze statisticamente significative su tutti e tre i confronti diretti.

L'analisi sui costi per interventi sanitari non-farmacologici non evidenzia differenze significative fra i gruppi (Tabella VII) anche se, tendenzialmente, dai valori medi rilevati, l'uso

\begin{tabular}{lcccc}
\hline & $\begin{array}{c}\text { CGI-gravità } \\
\text { T0 }\end{array}$ & $\begin{array}{c}\text { CGI-gravità } \\
\text { Tf }\end{array}$ & $\begin{array}{c}\text { Differenza } \\
\text { Tf - T0 }\end{array}$ & $\begin{array}{c}\text { p-value } \\
\text { Tf - T0 }\end{array}$ \\
\hline Olanzapina & 4,57 & 4,33 & $-0,24$ & $<0,001$ \\
Risperidone & 4,61 & 4,60 & $-0,01$ & 0,874 \\
Tipici & 4,25 & 4,37 & 0,12 & 0,182 \\
Significatività: & & & & \\
O-R & & & $\mathrm{p}=0,0018$ & \\
O-T & & & $\mathrm{p}<0,0001$ & \\
R-T & & $\mathrm{p}=0,1262$ & \\
\hline
\end{tabular}

Tabella IV

Punteggio medio CGI-gravità per gruppo di terapia: differenze fra TO e Tf

$\mathrm{O}=$ Olanzapina; $\mathrm{R}=$ Risperidone; $\mathrm{T}=$ Tipici

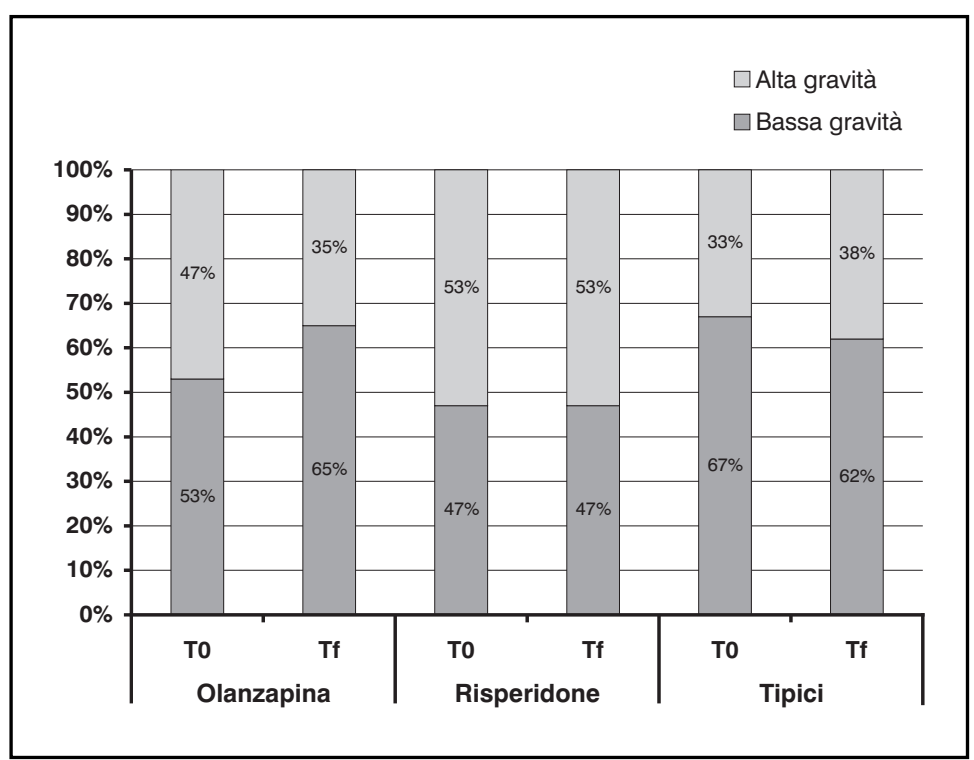

Figura 2

Percentuale di pazienti appartenenti ai cluster "alta" e "bassa" gravità ai tempi TO e Tf

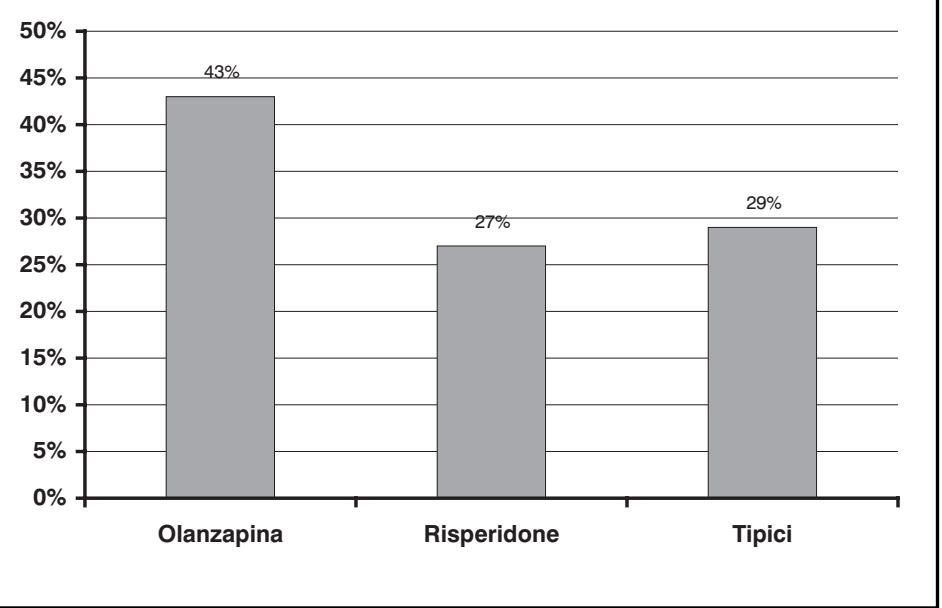

Figura 3

\% di pazienti con effetto terapeutico da "moderato" a "notevole" senza alcun effetto collaterale riscontrato 


\section{Tabella V}

Punteggio medio PANSS ai tempi TO eTf per gruppo di terapia

*Non si riscontrano differenze statisticamente significative nei confronti diretti fra i tre

\begin{tabular}{|c|c|c|c|c|}
\hline & TO & Tf & Delta Tf-TO* & p-value \\
\hline \multicolumn{5}{|l|}{ Scala positiva: } \\
\hline Olanzapina & 22,25 & 19,66 & $-2,59$ & $<0,001$ \\
\hline Risperidone & 22,35 & 20,79 & $-1,56$ & $<0,001$ \\
\hline Tipici & 19,35 & 17,96 & $-1,39$ & 0,123 \\
\hline \multicolumn{5}{|l|}{ Scala negativa: } \\
\hline Olanzapina & 27,47 & 23,82 & $-3,65$ & $<0,001$ \\
\hline Risperidone & 28,61 & 25,52 & $-3,09$ & $<0,001$ \\
\hline Tipici & 24,96 & 22,40 & $-2,56$ & 0,002 \\
\hline \multicolumn{5}{|c|}{ Scala di psicopatologia generale: } \\
\hline Olanzapina & 55,42 & 49,44 & $-5,98$ & $<0,001$ \\
\hline Risperidone & 55,56 & 50,68 & $-4,88$ & $<0,001$ \\
\hline Tipici & 49,38 & 45,56 & $-3,82$ & 0,020 \\
\hline \multicolumn{5}{|c|}{ Punteggio totale: } \\
\hline Olanzapina & 105,15 & 92,92 & $-12,23$ & $<0,001$ \\
\hline Risperidone & 106,52 & 96,99 & $-9,53$ & $<0,001$ \\
\hline Tipici & 93,69 & 85,92 & $-7,77$ & 0,013 \\
\hline
\end{tabular}

di olanzapina sembrerebbe consentire una riduzione della spesa del $22 \%$ rispetto a risperidone e del $40 \%$ rispetto ai tipici. Ciò è imputabile al fatto che al trattamento con risperidone sono associati maggiori costi di ospedalizzazione mentre l'utilizzo dei tipici comporta un maggior consumo di risorse sanitarie territoriali (Tabella VII).

La valutazione dei costi totali di trattamento (farmaci + interventi sanitari) mostra in sintesi che il maggior costo di acquisto per farmaci rilevato nei gruppi atipici è quasi totalmente compensato dal minor ricorso all'utilizzo di al- tre risorse sanitarie rispetto ai neurolettici tipici (Tabella VIII).

I dati evidenziati in Tabella VIII non mostrano differenze statisticamente significative fra i gruppi di trattamento in termini di costo totale medio annuo a paziente.

Il periodo biennale di osservazione dello studio ci ha permesso di valutare le differenze di costo fra i tre gruppi a confronto, rilevate in termini di trend tra l'anno I e l'anno II. La Tabella IX evidenzia olanzapina come il farmaco in grado di generare riduzioni significative, fra l'anno I e l'anno II, di tutte le

\footnotetext{
Tabella VI

Costi farmacologici medi annui a paziente per tipo di terapia (dati rilevati nell'anno /I costi in euro)

$\mathrm{O}=$ Olanzapina

$\mathrm{R}=$ Risperidone;

$\mathrm{T}=$ Tipici
}

\begin{tabular}{lccccc}
\hline & Neurolettici & Antidepressivi & $\begin{array}{c}\text { Stabilizzatori } \\
\text { umore }\end{array}$ & Anticolinergici & Totale farmaci \\
\hline Olanzapina & 1.980 & 85 & 34 & 2 & 2.100 \\
Risperidone & 1.438 & 94 & 33 & 5 & 1.571 \\
Tipici & 247 & 154 & 39 & 21 & 462 \\
Significatività: & & & & & $p<0,0001$ \\
O-R & $p=0,0006$ & $p=0,7451$ & $n s$ & $p=0,1217$ & $p<0,0001$ \\
O-T & $p<0,0001$ & $p=0,0317$ & $n s$ & $p<0,0001$ & $p<0,0001$ \\
R-T & $p<0,0001$ & $p=0,0786$ & $n s$ & $p<0,0001$ & $p$ \\
\hline
\end{tabular}


T. Federico, C. Astuto, G. Bongiorno, D. Majorana, G. Biricolti, S. Zerbo

voci di costo associate al trattamento. Unica eccezione la variazione della spesa per farmaci che, in termini tendenziali, vede comunque olanzapina l'unica terapia in grado di ridurre questa voce di costo (Tabella IX). Ciò è probabilmente imputabile al miglioramento delle condizioni generali dei pazienti del gruppo olanzapina, come testimoniato dall'andamento del punteggio totale CGI (Tabella IV).

Invece l'incremento, tra anno I e II, dei costi di trattamento farmacologico con tipici e risperidone riflette probabilmente la necessità di aggiustamenti di dose e associazione.

L'analisi delle differenze dei costi totali di trattamento fra I e II anno di osservazione evidenzia infine differenze statisticamente significative $(p=0,0104)$ solo per il confronto olanzapina ( -724 euro) vs. risperidone ( +373 euro) (Tabella IX). Tale andamento viene confermato dalla Figura 4 in cui sono state rappresentate le stesse differenze espresse in termini percentuali.

\section{Valutazioni di costo/efficacia}

In ambito farmacoeconomico le tecniche cosiddette "complete" sono quelle che valutano due o più trattamenti sanitari non solo sul versante dei costi ma anche su quello dei risultati di efficacia $[4,17]$.

Queste tecniche di analisi, dette anche di "costo/efficacia", vengono di norma utilizzate quando, in contesti di limitate disponibilità di

\begin{tabular}{lccc}
\hline & Ricoveri & $\begin{array}{c}\text { Risorse } \\
\text { sanitarie territoriali* }\end{array}$ & $\begin{array}{c}\text { Totale } \\
\text { interventi sanitari** }\end{array}$ \\
\hline Olanzapina & 110 & 1.620 & 1.729 \\
Risperidone & 463 & 1.747 & 2.210 \\
Tipici & 314 & 2.582 & 2.896 \\
\hline
\end{tabular}

Tabella VII

Costo medio annuo a paziente per interventi sanitari non-farmacologici (dati riferiti all'anno II - costi in euro)

* Visite specialistiche, interventi medici/infermieristici domiciliari e in struttura, frequenza centro diurno, interventi di psicoterapia e di riabilitazione

** Non si riscontrano differenze statisticamente significative nei confronti diretti fra i tre trattamenti

\begin{tabular}{lccc}
\hline & $\begin{array}{c}\text { Costo } \\
\text { farmaci }\end{array}$ & $\begin{array}{c}\text { Costo } \\
\text { interventi sanitari }\end{array}$ & $\begin{array}{c}\text { Costo totale } \\
\text { di trattamento }\end{array}$ \\
\hline Olanzapina & 2.100 & 1.729 & 3.829 \\
Risperidone & 1.571 & 2.210 & 3.781 \\
Tipici & 462 & 2.896 & 3.358 \\
Significatività: & & & \\
O-R & $p<0,0001$ & $n \mathrm{~ns}$ & $\mathrm{~ns}$ \\
O-T & $\mathrm{p}<0,0001$ & $\mathrm{~ns}$ & $\mathrm{~ns}$ \\
R-T & $\mathrm{p}<0,0001$ & $\mathrm{~ns}$ & $\mathrm{~ns}$ \\
\hline
\end{tabular}

\section{Tabella VIII}

Costo di trattamento totale medio annuo a paziente per tipo di terapia (dati riferiti all'anno II - costi in euro)

$\mathrm{O}=$ Olanzapina; $\mathrm{R}=$ Risperidone; $\mathrm{T}=$ Tipici

\begin{tabular}{|c|c|c|c|c|}
\hline & Olanzapina & Risperidone & Tipici & \\
\hline Farmaci (costo medio annuo a paziente anno II) & 2.100 & 1.571 & 462 & \\
\hline Differenza media a paziente vs anno I & -129 & 64 & 66 & \\
\hline$p$ value & ns & ns & ns & \\
\hline Interventi sanitari (costo medio annuo a paziente anno II) & 1.729 & 2.210 & 2.896 & \\
\hline Differenza media a paziente vs anno I & -595 & 309 & -82 & \\
\hline$p$ value & 0,0289 & ns & ns & \\
\hline Ricoveri (costo medio annuo a paziente anno II) & 110 & 463 & 314 & \multirow{7}{*}{$\begin{array}{l}\text { Tabella IX } \\
\text { Costi di trattamento } \\
\text { medi annui a paziente } \\
\text { rilevati nell'anno I/ e } \\
\text { variazioni rispetto } \\
\text { all'anno I (costi in euro) }\end{array}$} \\
\hline Differenza media a paziente vs anno I & -439 & 206 & -143 & \\
\hline$p$ value & 0,0094 & ns & ns & \\
\hline Totale trattamento (costo medio annuo a paziente anno II) & 3.829 & 3.781 & 3.358 & \\
\hline Differenza media a paziente vs anno I $^{*}$ & -724 & 373 & -15 & \\
\hline$p$ value & 0,0083 & ns & ns & \\
\hline $\begin{array}{l}\text { * Significatività: } \\
\text { O-R } \\
\text { O-T } \\
\text { R-T }\end{array}$ & $\begin{array}{c}\mathrm{p}=0,0104 \\
\mathrm{~ns} \\
\mathrm{~ns}\end{array}$ & & & \\
\hline
\end{tabular}




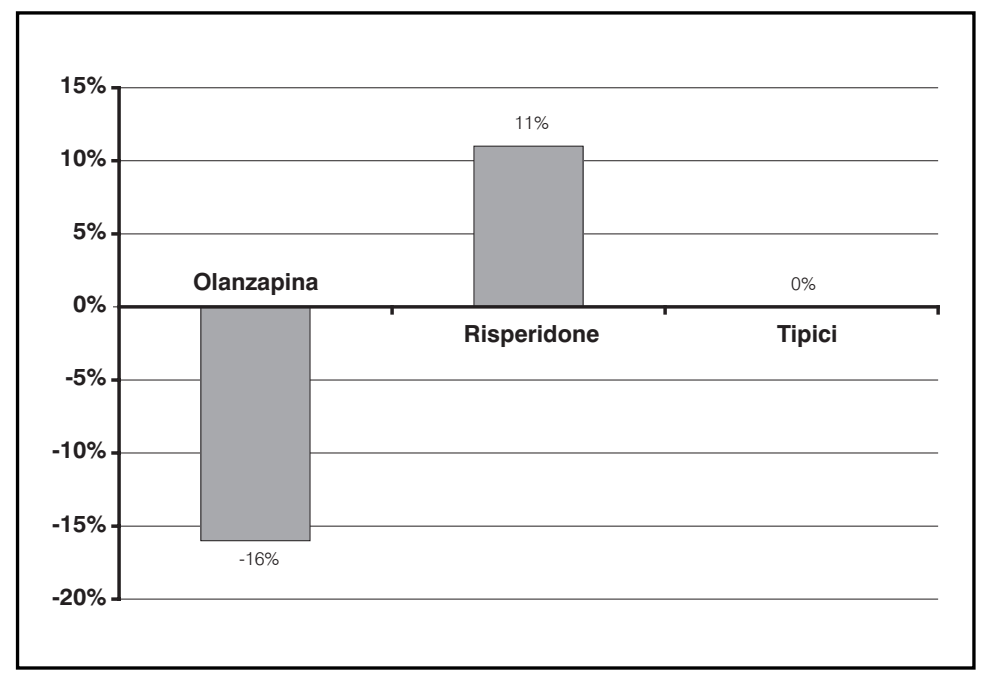

Figura 4

Costo totale medio annuo a paziente per tipo di terapia: variazioni \% fra I e II anno di osservazione

$\mathrm{P}<0,05$ nel confronto olanzapina-risperidone

risorse, si renda necessario operare delle scelte tra programmi terapeutici alternativi.

In tali circostanze si fa spesso riferimento al calcolo del rapporto medio costo/efficacia, ordinando i diversi programmi sanitari a confronto in base al relativo valore scaturito da tale rapporto.

Successivamente, sulla base dell'ammontare delle risorse disponibili, verrà scelto il trattamento più efficiente ovvero che garantisca la migliore efficacia al costo più basso [16].

Nell'ambito del presente lavoro si è cercato di seguire tale approccio con l'obiettivo di fornire supporto all'individuazione delle terapie antipsicotiche più efficienti e che consentano una allocazione ottimale delle risorse, sempre più scarse nel contesto del panorama sanitario nazionale.

Ai fini del calcolo del rapporto medio costo/efficacia per i tre trattamenti a confronto, si è utilizzato come valore di costo (numeratore del suddetto rapporto) il "costo totale medio di trattamento" (Tabella VIII).

Come indicatore di efficacia (denominatore del rapporto) si è invece optato per la riduzione del punteggio di gravità-CGI tra $\mathrm{T} 0$ e Tf nell'anno II di osservazione (Tabella IV).
In Tabella $\mathrm{X}$ vengono riportati, per tipo di terapia, i valori di "costo medio per punto di gravità ridotto su scala CGI". Tale parametro non è valutabile per i neurolettici tipici in quanto per questo tipo di trattamento si è riscontrato un aumento, anziché una diminuzione, del punteggio medio di gravità-CGI fra T0 e Tf e conseguentemente risulta non identificabile il denominatore del relativo rapporto medio di costo/efficacia. Per quanto riguarda invece il risultato di risperidone abbiamo calcolato comunque il rapporto medio di costo/efficacia nonostante le differenze al denominatore non fossero statisticamente significative (Tabella IV).

\section{DISCUSSIONE}

Il valore di un trattamento antipsicotico può essere misurato in vari modi; tra i principali fattori che vengono presi in considerazione a tale fine, oltre al versante dei costi, ci sono la risposta al trattamento misurata in termini di miglioramento o peggioramento della gravità, la compliance al trattamento, il miglioramento dei sintomi (positivi, negativi, etc.) e la presenza o meno di effetti collaterali $[5,18]$.

In questo lavoro si è adottato un approccio di tipo osservazionale su pazienti normalmente afferenti a Strutture psichiatriche territoriali al fine di ottenere una valutazione farmacoeconomica dei trattamenti il più possibile aderente alla reale pratica clinica. La casualità nella composizione dei gruppi è stata ottenuta con un criterio di arruolamento in successione temporale secondo l'entrata in contatto dei pazienti con le strutture psichiatriche. Non si è potuto adottare il classico metodo della randomizzazione per codice dal momento che lo studio ha seguito metodi naturalistici. In tale ambito si è proceduto a confrontare tre terapie antipsicotiche (olanzapina, risperidone, neurolettici tipici) sia sul versante economico che su quello dell'efficacia clinica.

Sul piano dei costi è stato valutato il consumo di risorse sanitarie associato ai tre trattamenti includendo nell'analisi il costo dei farmaci, le ospedalizzazioni e le principali pre-

\section{Tabella $\mathrm{X}$}

Costo medio per punto di gravità ridotto su scala CGI (costi in euro)

\begin{tabular}{lccc}
\hline & Olanzapina & Risperidone & Tipici \\
\hline Costo totale medio di trattamento a paziente & 3.829 & 3.781 & 3.358 \\
Punteggio medio CGl rilevazione To & 4,57 & 4,61 & 4,25 \\
Punteggio medio CGl rilevazione Tf & 4,33 & 4,60 & 4,37 \\
Differenza media di punteggio rilevazioni Tf-To & $-0,24$ & $-0,01$ & 0,12 \\
Costo medio per punto di gravità ridotto su scala CGl & 15.954 & 378.100 & $\mathrm{nv}$ \\
\hline
\end{tabular}


stazioni psichiatriche territoriali a carico del SSN (visite specialistiche, interventi medici/ infermieristici, interventi di psicoterapia, di riabilitazione, frequenza del centro diurno).

La misurazione dell'efficacia delle terapie è stata invece effettuata attraverso l'utilizzo delle scale di valutazione psichiatrica CGI (Clinical Global Impression) e PANSS (Positive and Negative Syndrome Scale) [11].

La scarsa numerosità dei pazienti inclusi nel gruppo dei neurolettici tipici (dovuta peraltro al frequente utilizzo delle formulazioni depot che, come requisito di arruolamento, sono state escluse dallo studio) rispetto ai gruppi degli atipici, unita alla non inclusione in analisi di altri trattamenti antipsicotici oggi diffusi in psichiatria potrebbero rappresentare potenzialmente dei bias per il nostro studio, fornendo un quadro parziale di valutazione farmacoeconomica dei trattamenti antipsicotici.

Inoltre i risultati emersi da questo lavoro si riferiscono ad una realtà psichiatrica locale; rimane quindi tutto da dimostrare che le conclusioni a cui si è giunti siano estendibili all'intero panorama psichiatrico nazionale.

In ogni caso sul piano clinico lo studio evidenzia olanzapina come il farmaco con il miglior profilo di efficacia. Questa molecola ha infatti denotato vantaggi statisticamente significativi rispetto agli altri trattamenti in termini di riduzione di punteggio medio di gravità-CGI (Tabella IV); a questo risultato si associa la migliore performance come "indice di efficacia - CGI" che vede olanzapina al primo posto come effetto terapeutico combinato con l'assenza di effetti collaterali severi (Figura 3). Tali aspetti sono inoltre confermati da una migliore attitudine a trasferire maggiori percentuali di pazienti da fasce di elevata gravità a fasce di bassa gravità CGI (Figura 2).

Le rilevazioni effettuate attraverso la scala PANSS non hanno denotato scarti statistica- mente significativi fra i gruppi anche se, tendenzialmente, le differenze fra terapie in termini di riduzione media del punteggio di gravità confermano i risultati evidenziati con la scala CGI (Tabella V).

Sul piano economico invece i risultati dello studio stanno ad indicare che il costo di acquisto dei farmaci è solo una componente del costo totale sostenuto dal Sistema Sanitario per il trattamento del paziente psicotico. I dati confermano che, con l'utilizzo degli atipici, al maggior costo di acquisto del farmaco corrisponde un minor consumo delle altre risorse sanitarie. Questo aspetto emerge chiaramente dalla Tabella VIII che evidenzia l'equivalenza, in termini statistici ( $p>0,05$ su tutti i confronti diretti), del costo totale medio di trattamento fra i tre gruppi analizzati.

Il trend fra I e II anno di osservazione evidenzia inoltre olanzapina come l'unica scelta in grado di ridurre in maniera statisticamente significativa $(\mathrm{P}=0,0083)$ il costo totale medio di trattamento del paziente psicotico (Tabella IX) nonostante partisse, il primo anno di osservazione, da livelli di costo più elevati sebbene non statisticamente separabili da quelli degli altri gruppi. Questo risultato emerso dalla ricerca, oltre ad evidenziare un impiego più efficiente delle risorse collegato all'uso di olanzapina, conferma quanto rilevato sulle scale CGI e PANSS in termini di migliore performance clinica e risocializzante, a lungo termine, sul paziente psicotico.

La riduzione media di costo totale a paziente per olanzapina fra I e II anno di osservazione (-724 euro) denota inoltre un vantaggio statisticamente significativo $(p=0,0104)$ verso risperidone che invece fa rilevare un incremento medio a paziente sul costo totale di trattamento (+373 euro). Livelli costanti di costo totale medio di trattamento si sono rilevati invece nel gruppo trattato con neurolettici tipici (Tabella IX, Figura 4).

La migliore performance di efficacia clinica e di minore incidenza di effetti collaterali, as-

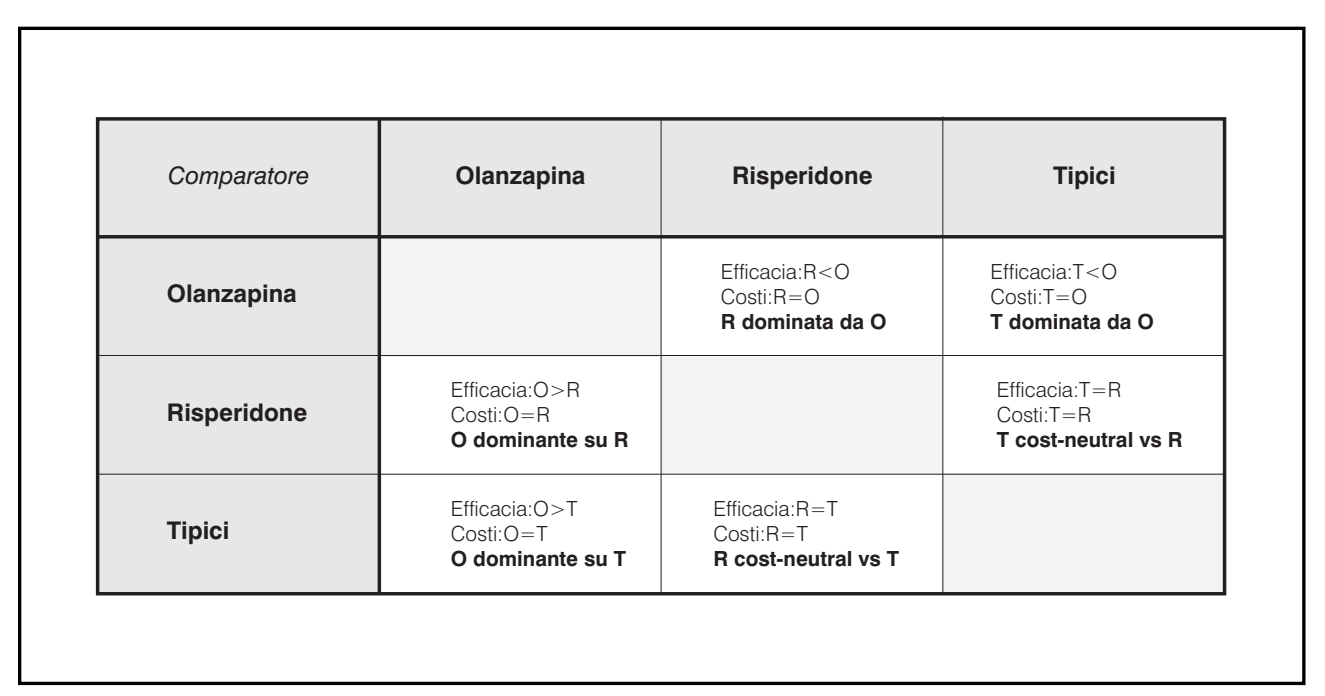

Figura 5

Sintesi dei risultati dell'analisi farmacoeconomica

$\mathrm{O}=$ Olanzapina; $\mathrm{R}=$ Risperidone $\mathrm{T}=$ Tipici 
sociata con l'affidabilità a lungo termine sul piano economico sembrano dunque essere alla base del migliore profilo farmacoeconomico per olanzapina nell'ambito delle terapie antipsicotiche confrontate nella realtà psichiatrica catanese.

Anche se, per i motivi sopra descritti, rimane difficile arrivare a delle ferme conclusioni, la Figura 5 sintetizza i risultati dello studio soffermandosi sui confronti diretti tra far- maci analizzati da un punto di vista farmacoeconomico.

In virtù della maggiore efficacia clinica e dell'equivalenza sul piano dei costi totali di trattamento, olanzapina risulta la scelta "dominante" sia verso risperidone che verso i neurolettici tipici.

Il trattamento con risperidone risulta invece "cost-neutral" rispetto a quello con i tipici alla luce dell' equivalenza fra le due terapie sia sul piano clinico che su quello economico.

\section{BIBLIOGRAFIA}

1. Worrel J. A., Marken P.A., Beckman S.E., Ruther V.. L., Atypical antipsychotic agents: A critical review , Am J Health-Syst Pharm vol 57, 238-255

2. Del Paggio D. et al. Clinical and economic outcomes associated with olanzapine for the treatment of psychotic symptons in a County Mental Health population, Clin Ther, 2002; 24: 803-817

3. Edgell E.T. et al. Olanzapine versus risperidone - A prospective comparison of clinical and economic outcomes in schizophrenia, Pharmacoeconomics, 2000, Dec; 18 (6): 567-579

4. Mapelli V. et al. Costi ed efficacia della cura della schizofrenia con antipsicotici tipici ed atipici, PharmacoEconomics Italian Research Articles, 2003; vol. 5, n. 3, 1-15

5. Ravasio R. La valutazione economica del trattamento farmacologico con antipsicotici nella schizofrenia: una revisione sistematica della letteratura, Farmeconomia e percorsi terapeutici, 2005; 6 (1): 25-36

6. Rollnik JD et al. Short-term cognitive improvement in schizophrenics treated with typical and atypical neuroleptics, Neuropsychobiology 2002; 45 (2): 74-80

7. Le tecniche farmacoeconomiche, Sezione Rubriche, Farmeconomia e percorsi terapeutici, 2000; 1(2): 122-124

8. Hamilton S.H. et al. Clinical and economic outcomes of olanzapine compared with haloperidol for schizophrenia, Pharmacoeconomics, 1999, may; 15 (5): 469-480

9. Garattini L. et al. ASI, 1996, n. 10: 33-37 
10. www.provincia.catania.it

11. Conti L.; Repertorio delle scale di valutazione in Psichiatria, tomo primo; See-Firenze; 1999; 197-207

12. The ICD-10 Classification of Mental and Behavioural Disorders, World Health Organization, Geneva, 1992; National electronic Library of Health - Mental Health

13. Drummond M.F. et al. Metodi per la valutazione economica dei programmi sanitari, Il Pensiero Scientifico Editore, 2000:21-30

14. Garattini L., Rossi C., Tediosi F. et al. Costi diretti della schizofrenia nei dipartimenti di salute mentale italiani. Pharmacoeconomics Italian Research Articles, 2002; 4(2): 45-95

15. L'Informatore farmaceutico, $2004, \mathrm{OEMF}, 65^{\circ}$ edizione

16. Filippelli E. et al. Il trattamento dei disturbi psicotici con olanzapina, risperidone e neurolettici tipici: una valutazione di costo/efficacia in una realtà psichiatrica locale, Farmeconomia e percorsi terapeutici, 2005; 6(3): 161-168

17. Duggan L., Fenton M., Dardennes R.M., El-Dosoky A., Indran S.; Olanzapine for schizophrenia; Cochrane Library (1);2000; $1-81$

18. Almond S. et al. Cost analysis of the treatment of schizophrenia in the UK: a comparison of olanzapine, risperidone and haloperidol, Pharmacoeconomics 1998; 13: 575-588 